\title{
CONCERNING NEW PERTURBATION METHODS IN SOLAR SYSTEM DYNAMICS
}

\author{
E. A. GREBENIKOV \\ Institute of High-Performance Computing Systems \\ Moscow, Russia
}

\section{Introduction}

In this paper a new method of construction of the perturbation motion theory of celestial bodies, based on the averaging principle in view of frequency resonances, is stated. The first approximation of the asymptotic theory is the exact solution of the dynamics averaging equations, in which are included "secular" and "long-periodic" terms. The high-degree approximations are the exact solution of a known Krylov-Bogoliubov generalized equation. It is shown that these iterations are expressed in the analytical form by multiple Fourier series.

\section{New Variants of the Perturbation Theory}

Let us consider a $n$-dimensional differential equation with small parameter $\mu$,

$$
\frac{d z}{d t}=Z(z, t, \mu), \quad z(0)=z_{0}
$$

where the vector-function $Z(z, t, \mu)$ is determined, and has properties, guaranteeing the existence and uniqueness of the solution of the Cauchy problem (1) in the $(n+1)$-dimensional domain $G_{(n+1)}=\{z \in G \times R \ni t\}$ of the Euclidean space.

Our purpose is to construct this solution. Together with equation (1) we consider an equivalent one,

$$
\frac{d z}{d t}=\bar{Z}(z, t, \mu)+Z(z, t, \mu)-\bar{Z}(z, t, \mu), \quad z(0)=z_{0}
$$

in which $\bar{Z}(z, t, \mu)$ is an arbitrary function. We write a linear equality

$$
z(t, \mu)=\bar{z}(t, \mu)+u(\bar{z}, t, \mu)
$$

I. M. Wytrzyszczak, J. H. Lieske and R. A. Feldman (eds.),

Dynamics and Astrometry of Natural and Artificial Celestial Bodies, 399, 1997.

(C) 1997 Kluwer Academic Publishers. Printed in the Netherlands. 
where $\bar{z}, u$ are some new unknown functions.

Equality (3) represents the transformation from the phase space $\{z\}$ to the new phase space $\{\bar{z}\}(\{z\} \rightarrow\{\bar{z}\})$, and the inverse $(\{\bar{z} \rightarrow z\})$, if the Jacoby matrix $\left(\frac{\partial u}{\partial \bar{z}}\right)$ is nonsingular.

The following differential identity holds:

$$
\frac{d z}{d t} \equiv \frac{d \bar{z}}{d t}+\left(\frac{\partial u}{\partial \bar{z}}, \frac{d \bar{z}}{d t}\right)+\frac{\partial u}{\partial t}
$$

where $\left(\frac{\partial u}{\partial \bar{z}}, \frac{d \bar{z}}{d t}\right)$ is a product of the matrix $\left(\frac{\partial u}{\partial \bar{z}}\right)$ and the vector $\frac{d \bar{z}}{d t}$.

The solution of the Cauchy problem (1) can be found by solving the following two Cauchy problems:

and

$$
\frac{d \bar{z}}{d t}=\bar{Z}(\bar{z}, t, \mu), \quad \bar{z}(0)=\bar{z}_{0} \in G_{n}
$$

$$
\frac{\partial u}{\partial t}+\left(\frac{\partial u}{\partial \bar{z}}, \bar{Z}(\bar{z}, t, \mu)\right)=Z(\bar{z}+u, t, \mu)-\bar{Z}(\bar{z}, t, \mu), \quad u(0)=z_{0}-\bar{z}_{0},
$$

where $\bar{z}_{0}$ is some new initial point. Equation (5) defines the choice of the initial appoximation $\bar{z}(t, \mu)$ for the exact solution $z(t, \mu)$ of problem (1) and equation (6) defines the total perturbation $u(\bar{z}, t, \mu)$. In classical perturbation theory, the function $u$ depends only on $t$ and $\mu$; therefore, instead of equation (6) we have:

$$
\frac{d u}{d t}=Z(\bar{z}+u, t, \mu)-\bar{Z}(\bar{z}, t, \mu), \quad u(0)=z_{0}-\bar{z}_{0} .
$$

For the Cauchy problem (1) it is possible to construct a set of variants of the perturbation theory with $\bar{Z}$ and $\bar{z}_{0}$. We call $\bar{Z}(\bar{z}, t, \mu)$ and $z_{0}$ generators of the perturbation theory for problem (1), and equation (5) a generating equation for equation (1).

In classical works on celestial mechanics linear generators are commonly used with respect to $\bar{z}$, and $\bar{z}_{0}=z_{0}$, that is,

$$
\begin{cases}\frac{d \bar{z}}{d t}=A(t) \bar{z}, & \bar{z}(o)=z_{0} \\ \frac{d u}{d t}=Z(\bar{z}+u, t, \mu)-A(t) \bar{z}, & u(0)=0\end{cases}
$$

or

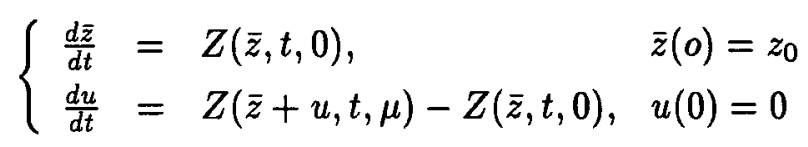

or

$$
\begin{cases}\frac{d \bar{z}}{d t}=\bar{Z}(\bar{z}, t, \mu), & \bar{z}(o)=z_{0} \\ \frac{d u}{d t}=Z(\bar{z}+u, t, \mu)-\bar{Z}(\bar{z}, t, \mu), & u(0)=0\end{cases}
$$


Equations (8) represent the linearisation method, equations (9) the method of small parameters, and equations (10) the averaging method, if the generator $\bar{Z}$ is constructed on the basis of some average operator.

The perturbation theory based on equations (5) and (6), differs from the classical perturbation theory in an essential point: the determination of perturbation $u(\bar{z}, t, \mu)$ from equation (6) does not require the preliminary solving of a generating equation (5). This fact permits one to determine the perturbations $u(\bar{z}, t, \mu)$ and the initial approximation of $\bar{z}(t, \mu)$ independently from each other. In the classical perturbation theory this is impossible. In Grebenikov (1986) and in Grebenikov and Mitropolskij (1992), we called equation (6) the generalized Krylov-Bogoliubov equation. Equation (6) is a Cauchy problem for the quasilinear $n$-dimensional system of partial differential equations of order 1 with respect to the $n$-dimensional perturbation vector $u(\bar{z}, t, \mu)$. Its solution can be found by the method of characteristics, or by the method of Cauchy (Stepanov, 1968).

\section{Differential Equations, Given on a Torus.}

A quite complete asymptotic theory of equation (6) for celestial mechanics problems was developed in monographs by Grebenikov (1986), and by Grebenikov and Mitropolskij (1992). We investigated the problems of dynamics, which are described by multifrequent systems of differential equations, given on a torus and, in particular, by Hamiltonian systems with "action angle" type variables and with a Hamiltonian, periodic in angular variables.

Let a problem of celestial mechanics be described by a multifrequent system of order $(m+n)$,

$$
\begin{cases}\frac{d x}{d t}=\mu X(x, y), & x(0)=x_{0}, \\ \frac{d y}{d t}=\omega(x)+\mu Y(x, y), & y(0)=y_{0},\end{cases}
$$

where $x, X$ are $m$-dimensional vectors, $y, Y, \omega$ are $n$-dimensional vectors, and $\omega(x)$ is a vector of frequences. We assume, that $X(x, y), Y(x, y)$ are $2 \pi$-periodic functions with respect to $y$. Then, they are represented by $n$ multiple Fourier series:

$$
\begin{aligned}
& X(x, y)=\sum_{\|k\| \in I} X_{k}(x) e^{i(k, y)}, \\
& Y(x, y)=\sum_{\|k\| \in I} Y_{k}(x) e^{i(k, y),}
\end{aligned}
$$

where $i=\sqrt{-1},(k, y)=\sum_{s=1}^{n} k_{s} y_{s},\|k\|=\sum_{s=1}^{n}\left|k_{s}\right|, I=\{0,1,2, \cdots\}$, and $k_{s}=0, \pm 1, \cdots$.

The equations of motion have such a form in planetary problems, as if the phase variables were Keplerian, Delauney's, Poincaré's etc. variables. 
We choose the generating system (5) in the form

$$
\left\{\begin{aligned}
\frac{d \bar{x}}{d t} & =\mu \bar{X}(\bar{x}, \bar{y})+\sum_{k \geq 2}^{N} \mu^{k} A_{k}(\bar{x}, \bar{y}), \\
\frac{d \bar{y}}{d t} & =\omega(x)+\mu \bar{Y}(\bar{x}, \bar{y})+\sum_{k \geq 2}^{N} \mu^{k} B_{k}(\bar{x}, \bar{y}),
\end{aligned}\right.
$$

where $\bar{X}, \bar{Y}, A_{k}, B_{k}$, are some arbitrary functions. Equations (13) are 'averaged equations of $N^{\text {th }}$ approximation' $(N=2,3, \cdots)$, and equations

$$
\frac{d \bar{x}}{d t}=\mu \bar{X}(\bar{x}, \bar{y}), \quad \frac{d \bar{y}}{d t}=\omega(x)+\mu \bar{Y}(\bar{x}, \bar{y})
$$

are 'average equations of first approximation'.

Let us consider the substitution (3) as formal series

$$
\left\{\begin{array}{l}
x=\bar{x}+\sum_{k \geq 1} \mu^{k} u_{k}(\bar{x}, \bar{y}) \\
y=\bar{y}+\sum_{k \geq 1}^{\sum} \mu^{k} v_{k}(\bar{x}, \bar{y})
\end{array}\right.
$$

with unknown functions $u_{k}(\bar{x}, \bar{y}), v_{k}(\bar{x}, \bar{y})$. To determine the functions of transformation $u_{k}$ and $v_{k}$, we have an infinite system of linear partial differential equations of first order

$$
\left\{\begin{aligned}
\left(\frac{\partial u_{1}}{\partial \bar{y}}, \omega(\bar{x})\right)= & X(\bar{x}, \bar{y})-\bar{X}(\bar{x}, \bar{y}) \\
\left(\frac{\partial v_{1}}{\partial \bar{y}}, \omega(\bar{x})\right)= & \left.\left(\frac{\partial \omega}{\partial \bar{x}}, u_{1}\right)\right)+Y(\bar{x}, \bar{y})-\bar{Y}(\bar{x}, \bar{y}), \\
\left.\frac{\partial u_{k}}{\partial \bar{y}}, \omega(\bar{x})\right)= & F_{k}\left(\bar{x}, \bar{y}, u_{1}, v_{1}, \cdots, v_{k-1}, u_{k-1}, A_{2}, B_{2}, \cdots, A_{k}\right), \\
\left.\frac{\partial v_{k}}{\partial \bar{y}}, \omega(\bar{x})\right)= & \Psi_{k}\left(\bar{x}, \bar{y}, u_{1}, v_{1}, \cdots, v_{k-1}, u_{k}, A_{2}, B_{2}, \cdots, A_{k}, B_{k}\right), \\
& k=2,3, \cdots
\end{aligned}\right.
$$

The system (15) has a remarkable property: it is possible to integrate it in analytical form (see Grebenikov and Ryabov, 1983; Grebenikov, 1986) for any vector-index $k$, if we choose for $\bar{X}$ and $\bar{Y}$ some averages of functions $X$ and $Y$. Let

$$
\begin{aligned}
& \bar{X}(\bar{x}, \bar{y})=\sum_{\|k\| \in I^{\prime}} X_{k}(\bar{x}) e^{i(k, \bar{y})}, \\
& \bar{Y}(\bar{x}, \bar{y})=\sum_{\|k\| \in I^{\prime}} Y_{k}(\bar{x}) e^{i(k, \bar{y})},
\end{aligned}
$$

where $I^{\prime} \in I$ is the subset of $I=\{0,1,2, \cdots\}$. In particular, $I^{\prime}$ can consist of one number - "zero", that means

$$
\bar{X}(\bar{x}, \bar{y})=X_{0}(\bar{x})=\frac{1}{(2 \pi)^{n}} \int_{0}^{2 \pi} \cdots \int_{0}^{2 \pi} X(\bar{x}, y) d y_{1} \cdots d y_{n}
$$


It is possible to find (see Stepanov, 1968) the exact solution of the first equations (15):

$$
\begin{aligned}
u_{1}(\bar{x}, \bar{y}) & =\sum_{\|k\| \in I \backslash I^{\prime}} \frac{X_{k}(\bar{x})}{i(k, \omega(\bar{x}))} e^{i(k, \bar{y})}+\varphi_{1}(\bar{x}), \\
v_{1}(\bar{x}, \bar{y}) & =\sum_{\|k\| \in I \backslash I^{\prime}} \frac{Y_{k}(\bar{x})}{i(k, \omega(\bar{x}))} e^{i(k, \bar{y})}+\left(\frac{\partial \omega(\bar{x})}{\partial x}, \sum_{\|k\| \in I \backslash I^{\prime}} \frac{X_{k}(\bar{x}) e^{i(k, \bar{y})}}{i^{2}(k, \omega(\bar{x}))^{2}}\right) \\
& +\left(\left(\frac{\partial u_{1}}{\partial \bar{x}}, \varphi_{1}(\bar{x})\right), \bar{y}\right)+\psi_{1}(\bar{x}) .
\end{aligned}
$$

Here, $\varphi_{1}, \psi_{1}$ are arbitrary differentiable functions of the arguments $\bar{x}_{1}, \cdots$, $\bar{x}_{m}$.

From analytical expressions (18) one can see, that if $\varphi_{1} \neq 0$, then function $v_{1}$ will be growing similar to the linear function $t$, because $\bar{y} \sim t$. Hence, to have an "oscillatory", but not "rapidly growing" character for the perturbations $u_{1}, v_{1}, u_{2}, v_{2}, \cdots$, it is necessary that all functions obey

$$
\varphi_{k}(\bar{x}) \equiv 0, \quad \psi_{k}(\bar{x}) \equiv 0, \quad k=1,2, \cdots
$$

These equalities, in their turn, show that the "best" perturbation theory is obtained when equations (5) are solved with other initial conditions than equation (1). The new initial conditions $\left(\bar{x}_{0}, \bar{y}_{0}\right)$ are connected with $\left(x_{0}, y_{0}\right)$ with the help of functional equations

$$
\begin{aligned}
& x_{0}=\bar{x}_{0}+\mu u_{1}\left(\bar{x}_{0}, \bar{y}_{0}\right), \\
& y_{0}=\bar{y}_{0}+\mu v_{1}\left(\bar{x}_{0}, \bar{y}_{0}\right) .
\end{aligned}
$$

This is a second essential difference between the modern perturbations theory and the classical, in which it is difficult to choose the initial point $\bar{z}_{0}$.

If we construct the perturbation theory of the second order, we shall have

$$
\begin{aligned}
& \left(\frac{\partial u_{2}}{\partial \bar{y}}, \omega(\bar{x})\right)=F_{2}\left(\bar{x}, \bar{y}, u_{1}, v_{1}, A_{2}\right), \\
& \left(\frac{\partial v_{2}}{\partial \bar{y}}, \omega(\bar{x})\right)=\Psi_{2}\left(\bar{x}, \bar{y}, u_{1}, v_{1}, u_{2}, A_{2}, B_{2}\right) .
\end{aligned}
$$

These equations include arbitrary functions $A_{2}, B_{2}$ which will be choosen such that

$$
\int_{0}^{2 \pi} \cdots \int_{0}^{2 \pi} F_{2} d \bar{y}_{1} \cdots d \bar{y}_{n}=0, \quad \int_{0}^{2 \pi} \cdots \int_{0}^{2 \pi} \Psi_{2} d \bar{y}_{1} \cdots d \bar{y}_{n}=0 .
$$

These conditions guarantee the "oscillatory" character of $u_{2}$ and $v_{2}$, if the functions $\varphi_{2}$ and $\psi_{2}$ (by analogy with $\varphi_{1}$ and $\psi_{1}$ ) are also chosen identically equal to zero. This procedure can be continued for $k=3,4, \cdots$. 
The stated analytical algorithm means, that we consequently construct the substitution of variables

$$
(x, y) \rightarrow\left(\bar{x}_{1}, \bar{y}_{1}\right) \rightarrow\left(\bar{x}_{2}, \bar{y}_{2}\right) \rightarrow \cdots \rightarrow\left(\bar{x}_{s}, \bar{y}_{s}\right),
$$

where

$$
\begin{aligned}
& x_{s}=\bar{x}+\sum_{k=1}^{s} \mu^{k} u_{k}(\bar{x}, \bar{y}), \\
& y_{s}=\bar{y}+\sum_{k=1}^{s} \mu^{k} v_{k}(\bar{x}, \bar{y}),
\end{aligned}
$$

Naturally, for the final construction of the solution of initial equations (11) one should solve "the average equations" (13) with new initial conditions $\bar{x}_{s}(0), \bar{y}_{s}(0)$.

The analytical expressions for $u_{k}(\bar{x}, \bar{y}), v_{k}(\bar{x}, \bar{y})$ may be constructed with the help of a computer.

\section{Conclusions}

1. The subset $I^{\prime}$ should be chosen from condition

$$
\left|\left(k, \omega_{0}\right)\right|<\epsilon=O(\mu),
$$

where $\omega_{0}$ is the initial value of the frequent-vector $\omega\left(x_{0}\right) . I^{\prime}$ is the "resonance subset".

2. In classical perturbation theory "the small divisors" $\left(k, \omega_{0}\right)$ are constant. In the new theory the divisors $(k, \omega(\bar{x}))$ are variable, and do not stay small for a long time.

3 . In the new theory the accuracy of the perturbation $u(\bar{z}, t, \mu)$ does not depend on the accuracy of the first approximation.

\section{References}

Grebenikov, E.A.: 1986, Metod Usrednieniya v Prikladnych Zadachakh, Nauka, Moskva (in Russian).

Grebenikov, E.A. and Mitropolskij Ju.A.: 1992, Metod Usrednieniya w Issledovaniyakh Resonansnykh Sistem, Nauka, Moskva (in Russian).

Grebenikov, E.A. and Ryabov, Ju.A.: 1983, Constructive Methods in the Analysis of Nonlinear Systems, MIR, Moscow.

Stepanov, V.V.: 1968, Kurs Differenciyalnkh Uravnienij, Nauka, Moskva (in Russian). 